\title{
Effects of Computerized Patient Records on Patients
}

\author{
Beena Ullala Mata B N, Rameshwara G N, Naveen Kumar G \\ and Raghavendra B
}

\begin{abstract}
Computerized Patient Records (CPR) are computer-based documentations of all the meetings between a patient and a physician that contain the data of the various details about the patient, any clinical observations, investigations and diagnostic results, mode of treatment and the details about the follow-up of the patient by the physician. They are used in most of the hospitals to provide a quality patient care. It has both, positive and negative effects on the physicians as well as on the patients. This paper emphasizes only on the various positive and negative effects that the system has on the patients.
\end{abstract}

Keywords--- Computerized Patient Records, Clinical Observations, Diagnostic Results, Treatment, Patient and Physician

\section{INTRODUCTION}

$\mathrm{A}$ Computerized Patient Record (CPR) receives the details about the various personal information of the patient such as name, age, contact details, gender, etc., any past medical conditions or problems that have been treated or are still under treatment and medications and stores them as an encrypted code on the computer system thus, allowing the user to retrieve the data stored when the patient arrives with a different health problem.

These records accomplish many medical and business tasks efficiently to aid the doctors to provide a better patient care in a short duration of time. They provide an organized progression in patient data management systems which are being used in hospitals and in healthcare organizations [1]. These systems have both, positive effects as well as the negative effects on the patient in many ways as follows:

\section{Positive Effects}

- Availability of the medical data[2]

- Improved diagnosis and treatment[3]

- Reduction of cost[4]

- Safety of the patient[5]

Beena Ullala Mata B N, Associate Professor, Medical Electronics, BMSCE, Bangalore, India.E-mail:bnb.ml@bmsce.ac.in

Rameshwara G N, Student, Medical Electronics, BMSCE, Bangalore, India.E-mail:gnrameshwara@gmail.com

Naveen Kumar G, Student, Medical Electronics, BMSCE, Bangalore, India.E-mail:naveengnk07@gmail.com

Raghavendra B, Student, Medical Electronics, BMSCE, Bangalore, India. E-mail:raghavendra.b234@gmail.com

DOI: 10.9756/BIJMMI.8068

\section{Negative Effects}

- $\quad$ Security issues[6]

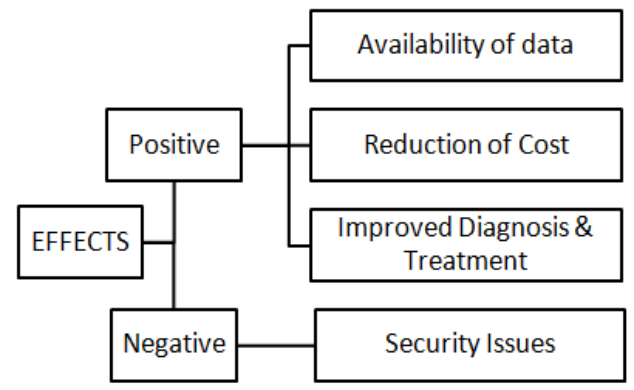

Figure 1: Different Effects of CPR on Patients

Each of the above effects of a CPR on the patient have been described in the following sections of this paper. section I describes about the effect of the availability of the medical data, section II describes about the effect of an improved diagnosis and treatment, section III describes about the effect of reduction of the cost, section IV describes about the effect of safety of the patient while section $\mathrm{V}$ describes about the various security issues that are faced and a solution to these issues.

Later, section B describes about the future scope of the CPR in hospitals and other healthcare organizations and the effects that it would have on the patients, section $C$ discusses the details about a survey that was carried out at BSN Memorial Hospital on CPR and its future scope, section D provides the conclusion that has been inferred from this paper, section E provides an acknowledgement and at the end, section F provides the references.

\section{A. Availability of the Medical Data}

A Computerized Patient Record provides the following features that enable an access of the medical data with an ease:

- Better organization of the data in the format of layouts and efficient searching in the various categories.

- Options for generating the output data in different formats.

Better Organization of the Data in the Format of Layouts and Efficient Searching in the Various Categories

The data which is stored in a Computerized Patient Record is structured, encrypted and organized in a monosemous fashion so that, the user can have a standalone system for:

- Displaying the stored data

- Entering a new data 
- Retrieval of the data in any order (chronological or non-chronological order)

- Create disease, condition or a problem specific data review formats.

Paper documentations suffer from a number of temporary restrictions like the data being fixed in a particular order in which they were recorded and saved. This is avoided in these systems by allowing the patients to access their medical data based on their own choice of interpretation of the data like visualizing it or hearing certain voice messages at any point of time.

\section{Options for Generating the Output Data in Different Formats}

Data can be presented to the users through different formats such as:

- System-generated voice recordings

- Pagers

- Email

- Online medical profiles

In an additional to these, the system allows to send an instruction to external micro-controller controlled devices such as automatic pill dispensers and insulin infusion pumps among many other devices. These devices would then carry out the desired action with help of certain commands or instructions from the clinician (physician). Finally, a number of one dimensional images can be transformed back into a single three dimensional image and a superimposed image as visualization for the surgeon. This allows the patient to access the data in the desired format or as per the desired application at the right time [2].

\section{B. Improved Diagnosis and Treatment}

Computerized Patient Record System is a conglomeration of the functionality of the following three systems:

- Clinical Decision-making System (CDS)

- Computerized Physician Order Entry (CPOE) System

- Health Information Exchange (HIE) System

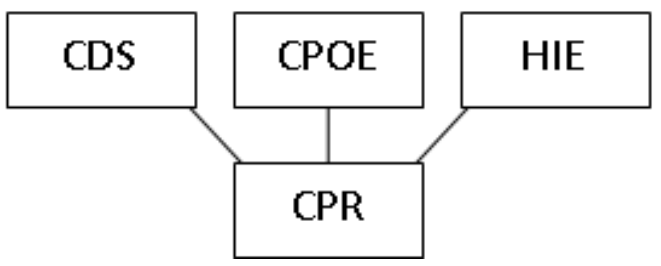

Figure 2: Functionalities of Computerized Patient Records

\section{Clinical Decision-making System (CDS)}

It is computer based system which aids the clinician to make a certain decision based on the clinical observations made by collecting the data given by the patient.

It allows the clinicians to take the various decisions with respect to patient care by providing an up-to-date information about the medications and treatments to which the patient is or may become allergic by showing undesired symptoms. It also generates audio or visual alerts when a certain potential problem is identified by the system. With an increasing growth of the medical information, each of the above functions provides decisions efficiently to safely deliver patient care. More the CDS systems are used, the chances of producing medical errors becomes less by being prevented by the computerized system. On an overall, the patient will receive a quality health care.

\section{Computerized Physician Order Entry (CPOE) System}

It is a computer-based system which receives the various orders of the patients from the clinician in order to eliminate all sorts of paper work such as filing, documenting, writing, etc.

It allows the clinicians to enter orders (regarding drugs, laboratory tests, any sort of therapy, etc.) into a computer rather than doing so on paper and thus minimizes the chances of occurrence of the potentially harmful medical errors that are caused by poor penmanship of the healthcare professionals (clinicians). Hence, this ensures a quality care to the patient.

\section{Health Information Exchange (HIE) System}

It is a computer-based system which enables the exchange of the medical data about a medical condition or problem of the patient between two or more healthcare organizations, two or more hospitals or between each other.

It allows the clinicians to transmit the patient information once it is electronically made to be available to other hospitals or healthcare organizations or within a group of authorized users. Thus, it provides an efficient patient care [3].

\section{Reduction of Cost}

Computerized Patient Records help in minimizing the inefficiency of paper-based processes and the wastes that they generate, thus eliminating all the paper work that is carried out for various documentations. This reduces the cost of filing the reports and scribbled notes about the health status of the patient. As this cost is charged by the clinicians as part of a nominal fee for diagnosing problems, the fee paid by the patient to the clinician is reduced.

These systems also help in reducing the costly redundant pre-tests that are suggested by the clinicians due to insufficient clinical information which is stored at another location. The cost for the clinical observation of the patient is thus reduced and hence, the patient would have to pay a reduced nominal amount rather than spending a large amount of money. Therefore, these systems assist in reduction of the cost of diagnosis effectively for the patients [4].

\section{Safety of the Patient}

Computerized Patient Records help in preventing various injuries to the patients that may be caused by the health care provided (that actually intends to help the patients) in the following ways:

- Whenever a medication is prescribed, the system automatically checks for the various problems that may arise due to the medication prescribed and different types of alerts such as visual alerts like LEDs, lights, etc. or audible alerts which include various types of sounds are used by the system for alerting the clinician when a problem has been detected due to the medication provided by 
maintaining a record of the medications or allergies that the patient already has or has acquired.

- Once the information is received, the system informs the clinician in the emergency department about any life-threatening conditions that might have arised, and emergency staff can adjust the care given to the patient appropriately, even when the patient is unconscious.

- When a problem occurs, the system exposes the potential harm that is caused to the patient to the clinicians so as to avoid more serious consequences for patients and provide suggestions to prevent these problems to the clinicians.

- They aid the clinicians to quickly and systematically identify problems and provide appropriate solutions [5].

\section{E. Security Issues}

The patient data stored in a Computerized Patient Record is susceptible to misuse and this would have a negative effect on the patients and also on their respective families. The harm caused may be a personal or a financial harm. For instance, the social security number of the patient can be used in an identity theft. Identity theft involving financial institutions has a significant effect on the public.

When a CPR without a well-defined security measures is used, patients lose their trust on the healthcare providers as a result of which they refuse to provide important medical information due to the unauthorized use of the information [6].

\section{Suggested Solutions}

- Use of audit trails to track authorized users who abuse the healthcare information stored on the CPR system by maintaining a record about each and every access and transactions that are made on the system.

- Use of login procedures which require the users to enter their passwords and user IDs as a minimal measure.

- Use of biometrics such as finger-prints, retinal scans, iris scans, voice recognition or sometimes even face recognition as a security measure for accessing the medical data of the patient which is stored in a profile.

- Use of smart cards which grant the access to the data stored only when a user produces the card with an authorized password.

- Maintenance of the integrity of the data stored, through the authentication of a user and the validity of content sent across a network. For an instance, Digital Signature Standard (DSS) can be used by employing cryptography technology.

- Use of coding systems and firewalls to protect the data and internal networks such as an intranet.

- Use of a well designed and tested backup scheme and disaster recovery system allows the system to become less prone to data loss [6].

\section{FUTURE SCOPE}

Computerized Patient Records have a scope for being launched under outpatient and inpatient settings. Once the system is setup, connectivity with the other systems is established to further improve the patient care. Investments in the online tools, such as portals that allow the patients to communicate with their physicians, to schedule appointments and access an integrated, up-to-date information about their health, can be made. This would offer the patients a secured access to their diagnostic results and medications.

The more advanced the system becomes, the more questions rise whether non-physicians can use these systems to diagnose and treat the patients without any involvement of the physician.

Thus, these systems can lead to a redefinition of the role of a physician, as they begin to perform the functions which were formerly being performed only by a physician. Thus, the future scope of CPR systems from the perspective of the patients, has an ever growing demand due to its ability to provide a cost-effective quality healthcare within a short duration of time [7].

\section{Hospital SURVEY}

A study on Computerized Patient Record Systems was carried out at BSN Memorial Hospital (now BMS Hospital), Bangalore on $5^{\text {th }}$ April, 2015.

The details about how these systems were employed in the hospital and the future scope of these systems were obtained. They are described below.

In this Hospital, CPRs were mainly used for:

- Computerized Radiography

- Hospital Management Information System

\section{Computerized Radiography}

The advanced models of the medical equipments used in the hospital, were provided with a software by the manufacturers who had designed these softwares in their own language for a desired working of the system. For processing, the device captured an image and transferred it to a computer called workstation in the desired format via an extension provided by the developers.

In the workstation, the software enables the physician to edit the image like, enlarging, cropping, enhancing, etc. that allowed a detailed study of the problem. Once the desired final image which is suitable for the analysis is obtained, it is converted into a format which is compatible for Digital Imaging and Communication(DICOM) in order to transfer the image by retaining its characteristics. Then the image is transferred as a DICOM image from one software to another for a detailed study by the doctor.

The specialized doctor uses another software to study the image carefully and analyze it with the help of already existing basic information about the anatomy, physiology and pathology of the organ which is affected and the problem that the organ is affected with. 


\section{Hospital Management Information System (HMIS)}

At present, the software which is used in the Computer based Patient Recording system at this hospital is,Sea.Doc.

Sea.Doc is a very versatile, user friendly system which comes as a basic module for small nursing, homes, hospitals or clinics and with plug in additional module that can be used by multi-speciality and super-speciality hospitals. It comes in many versions in order to suit the budgetary needs of almost any hospital. It has been designed using SQL and .NET computer programming languages.
The general features of this software are as follows:

- Multi-level security

- Control reporting on billing

- Operator wise reports

- Inventory control

- Bar coding

- Text messaging for notifying important information

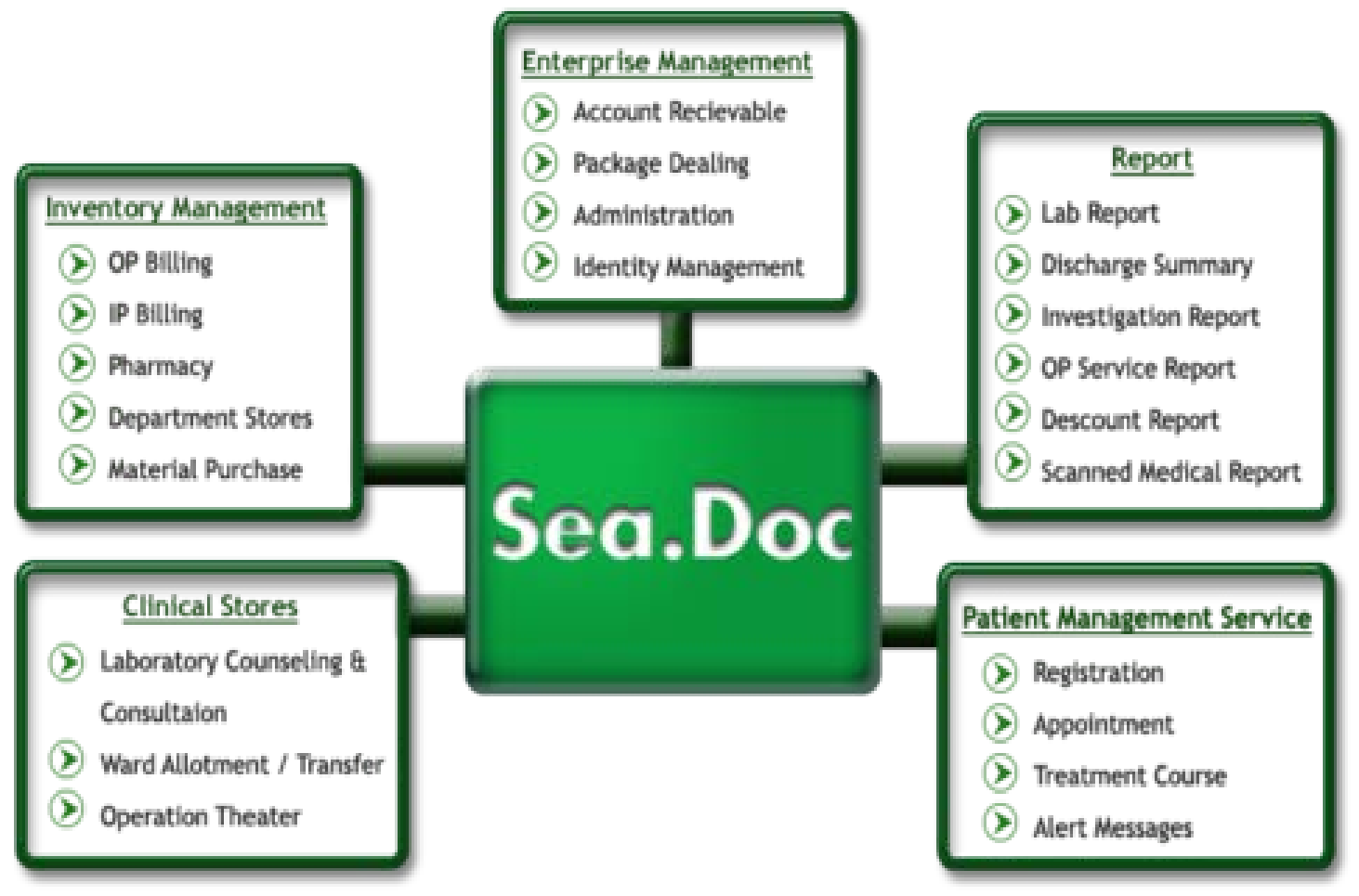

Figure 3: Functional Operations Offered by Sea.Doc software

The various functional operations offered by the software are summarized by figure 3 .

In the coming future, a patient who enters the hospital with a certain problem, does not have to carry any sort of files or scribbled notes but rather would be carrying a bluetooth enabled device and a smart card such as RFID card.

The RFID card would contain all the personal and medical details about the patient and allows him to access his medical profile where the data is stored. The bluetooth enabled device would be mounted with a sensor which can detect the basic physiological parameters like the heart rate, pulse rate, BP, etc. Thus, when the patient walks in and logs into his profile in the software using the RFID card, the patient can access all of the data that have been measured using the bluetooth device and are transferred into the software via bluetooth connection.

For reducing the tedious task of the diagnosing the problem, and for an easier analysis and diagnosis of the problem, the clinician would have the access the anatomy of essential organs of his specialization in relative to the problem being diagnosed.
The clinician then opens the anatomy, zooms it to study the structure in detail to allow him to analyze the problem and report it to the patient. The software would additionally show some of the common problems that can occur to that particular organ. The clinician is then supposed to select the problem and analyze it in order to diagnose the problem that is being described by the patient. Depending on the clinical complaints received and after an extensive study of the problem with help of the software, the problem is diagnosed. Once the problem is diagnosed, the software suggests possible medications that are later decided by the doctor to give to the patient.

\section{CONCLUSION}

Presently, the Computerized Patient Record Systems have a very less demand and applications due to their high initial and maintenance cost but, the promise which is offered by these systems for providing a cost-effective quality health care to the patient and the advancement in acquiring the medical knowledge through research is enormous from the perspective of the patient. 


\section{ACKNOWLEDGMENT}

We would like to thank Mr. Muralidhar from BSN Memorial Hospital, Bangalore for:

- Allowing us to carry out the study

- Giving us an enormous amount of valuable information about:

How CPRs are used in the hospital

The scope of these systems in the near future

\section{REFERENCE}

[1] R.D.Lele, "Computers in Medicine: Progress in Medical Informatics", 2005.

[2] Eddie Clinic, “Advantages of Computerized Medical Records”, Feb $22^{\text {nd }}, 2010$.

[3] Nir Menachemi and Taleah $\mathrm{H}$ Collum, "Benefits and drawbacks of Electronic Health Record Systems”, May $11^{\text {th }}, 2011$.

[4] Memorial Care Health Systems, "How Electronic Medical Records reduce costs and improve Patient outcomes”, July $12^{\text {th }}, 2010$.

[5] Healthit organization, "Improved Diagnostics and Patient outcomes".

[6] Software Engineering Ethics Research Institute (SEERI), "Security of Computerized Patient Record".

[7] Richard S Dick, Elaine B Steen and Don E Detmer, "The ComputerizedPatientRecord: An Essential Technology for Health Care”, $14^{\text {th }}$ Oct, 1997.

[8] Mohan Bansal, “Medical Informatics”, 2002.

[9] Richard S Dick, Elaine B Steen, "The computer-based Patient Records: An essential Technology for healthcare”, 1991.

[10] Elizabeth Mott, "Advantages and disadvantages of using a Computerized Patient Billing System"

[11] Vimla L Patel, Andre W Kushniruk, Seungmi Yang, Jean-Francois Yale, "Impact of a Computer-based Patient Record System on Data Collection, Knowledge Organization, and Reasoning, November, 2000.

[12] Harold P Lehman, Patricia A Abott, Nancy C Roderer, "Aspects of Computer based Patient Records”, 2013.

\section{ABOUt THE Authors}

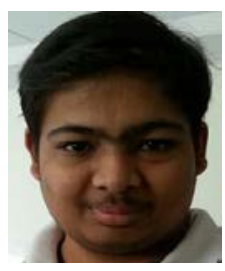

Rameshwara G N was born on $21^{\text {st }}$ November, 1994 at Bangalore. He completed his $10^{\text {th }}$ graduation at BNM Public School, Bangalore in 2010, PreUniversity at Vijaya Composite Pre-University College, Bangalore in 2012, and is now currently pursuing his Bachelor of Engineering in Medical Electronics at BMS College of Engineering, Bangalore.(E-mail: gnrameshwara@gmail.com)

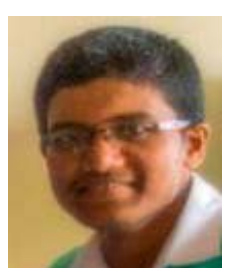

Naveen Kumar G was born on $28^{\text {th }}$ June, 1995 at Muddenahalli. He completed his $10^{\text {th }}$ graduation at Sri Sathya Sai LokaSeva High School, Muddenahalli in 2010, Pre-University at Sri Sathya Sai LokaSeva PreUniversity College, Muddenahalli in 2012, and is now currently pursuing his Bachelor of Engineering in Medical Electronics at BMS college of Engineering, Bangalore. (E-mail: naveengnk07@gmail.com)

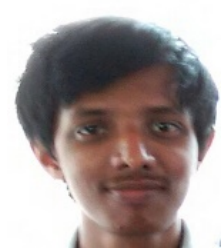

Raghavendra B was born on $29^{\text {th }}$ April, 1994 at Bangalore. He completed his $10^{\text {th }}$ graduation at Websters High School, Bangalore in 2010, PreUniversity at Sri BhagawanMahaveer Jain College, Bangalore in 2012 and is now currently pursuing his Bachelor of Engineering in Medical Electronics at BMS college of Engineering, Bangalore. (E-mail: raghavendra.b234@gmail.com) 